\title{
Mini Review on Anti-Tubercular Activity of Thioridazine on Basis of Molecular Docking Studies
}

\author{
Mohammad Asif ${ }^{*}$ and Md Tauquir Alam² \\ ${ }^{1}$ Department of Pharmaceutical Chemistry, Himalayan Institute of Pharmacy Research, Dehradun, India \\ ${ }^{2}$ Department of Pharmaceutical Chemistry, Faculty of Pharmacy, Northern Border University, Saudi Arabia \\ *Corresponding Author: Mohammad Asif, Department of Pharmaceutical Chemistry, Himalayan Institute of Pharmacy Research, \\ Dehradun, India.
}

Received: October 09, 2019; Published: October 14, 2019

DOI: $10.31080 /$ ASPS.2019.03.0418

\begin{abstract}
Thioridazine (TZ) is a drug that has been used for over 35 years as a psychoactive drug, is now potentially utilized in combination with certain anti-tubercular (anti-TB) drugs to cure Multi Drug Resistant (MDR), Extensively Drug Resistant (XDR) and Totally Drug Resistant (TDR) tuberculosis (TB). This study explores the possible reasons for its anti-TB activity by molecular docking procedure. Molecular docking studies were performed on seventeen enzymes from different mechanisms were docked to study the binding affinity of TZ on the active sites of various Mycobacterium tuberculosis (Mtb) enzymes in an effort to increase the understanding of the action of TZ as an anti-TB drug. The enzyme CmaA2-Cyclopropane mycolic acid synthase (PDB id: 1KPI) scored lowest binding energy which means the greater stability of the Thioridazine's ability to bind to the receptor. MmaA2 (1TPY), InhA (2NSD) and PknG (2PZI) enzymes of $M t b$ gave the best G-scores. The docking study results revealed that TZ may act by more than one possible mechanism to exert anti-tubercular activity against MDR-TB, XDR-TB and TDR-TB.
\end{abstract}

Keywords: Thioridazine; Molecular Docking; Enzymes; Binding Energy; MDR-TB; XDR-TB

\section{Introduction}

The World Health Organization (WHO) declared Tuberculosis (TB) is a global public health emergency in 1993 and it is still continues to be a major global health problem. Globally in 2012, an estimated 450,000 people developed Multi Drug Resistant (MDRTB) and there were an estimated 170,000 deaths from MDR-TB also there was reported cases of Extensively Drug Resistant (XDR) and Totally Drug Resistant (TDR) tuberculosis (TB). On an average, about $9.6 \%$ of MDR-TB cases have converted to XDR-TB [1]. A study of the use of Thioridazine (TZ) as salvage therapy in 4 Indian patients with XDR-TB (near total drug resistance to current therapy) with advanced disease. They found the drug to be done well tolerated, even in the malnourished and ill patient. It also led to clinical progress in 3 of the 4 patients [2]. The TZ, a neuroleptic drug, which is less toxic than chlorpromazine (CPZ), kills intracellular
$M t b$ isolates that are resistant to two or more antibiotics. The TZ shows anti-TB effects in-vitro and in-vivo mouse models [3]. The TZ is effective when used in combination with antibiotics to which the initial $M t b$ was resistant. Because TZ is cheap, it should be used in therapy of XDR-TB and TDR-TB patients in economically poor population $[4,5]$ and $\mathrm{TZ}$ in the treatment regimen on a 'compassionate' basis. The TZ in patients with XDR-TB who have exhausted all available drug options [6]. The TZ may be patented for its "new use". New drugs for treatment of TB, most notable MDR-TB and XDR-TB, are urgently needed; phenothiazines and their targets should be exploited for this use [7]. This study explore the possible mechanism of action of TZ by molecular docking studies. Though TZ was found to act through efflux pump mechanism, still it was not well explored [8]. 


\begin{tabular}{|c|c|c|c|}
\hline $\begin{array}{l}\text { S. } \\
\text { No. }\end{array}$ & Name of the enzyme (PDB ID) & $\begin{array}{c}\text { G-Score } \\
\text { (Kcal/mo) }\end{array}$ & Ref \\
\hline A & \multicolumn{3}{|l|}{ Function (Lipid Metabolism) } \\
\hline 1. & $\begin{array}{l}\text { CmaA-2 Cyclopropane Mycolic Acid } \\
\text { Synthase (1KPI) }\end{array}$ & -9.5 & {$[10]$} \\
\hline 2. & $\begin{array}{l}\text { MmaA2-Methoxy Mycolic Acid Syn- } \\
\text { thase } 2 \text { (1TPY) }\end{array}$ & -9.4 & {$[11]$} \\
\hline 3. & $\begin{array}{l}\text { InhA-NADH-Dependent Enoyl ACP } \\
\text { (Acyl Carrier Protein) Reductase } \\
\text { (2NSD) }\end{array}$ & -8.4 & [12] \\
\hline 4. & $\begin{array}{l}\text { FabH-(3-Oxo-acyl)-(Acyl Carrier } \\
\text { Protein) ACP Synthase (1HZP) }\end{array}$ & -3.7 & [13] \\
\hline 5. & $\begin{array}{l}\text { MbtK-Lysine-N(Epsilon)-acyltrans- } \\
\text { ferase (1YK3) }\end{array}$ & -2.2 & {$[14]$} \\
\hline 6. & $\begin{array}{l}\text { FabD-Malonyl CoA- Acyl Carrier } \\
\text { Protein transacylase (2QC3) }\end{array}$ & -3.8 & [15] \\
\hline 7. & $\begin{array}{l}\text { PcaA-Mycolic Acid (Cyclopropane) } \\
\text { Synthase (1L1E) }\end{array}$ & -5.4 & {$[16]$} \\
\hline 8. & $\begin{array}{l}\text { DprE1-Decaprenylphosphoryl-B-D- } \\
\text { Ribose 2'-Epimerase (4FD0) }\end{array}$ & -3.8 & {$[17]$} \\
\hline B & \multicolumn{3}{|c|}{ Function (Intermediary Metabolism and Respiration) } \\
\hline 9. & AdoK-Adenosine Kinase (2PKK) & -4.5 & [18] \\
\hline 10. & GlnA1-Glutamine Synthetase (4ACF) & -6.0 & {$[19]$} \\
\hline 11. & ThyX-Thymidylate Synthase (3GWC) & -2.0 & [20] \\
\hline 12 & TmK-Thymidylate Kinase (1G3U) & -3.8 & {$[21]$} \\
\hline C & \multicolumn{3}{|c|}{ Function (Cell wall and Cell Processes) } \\
\hline 13. & LdtB- L, D-Transpeptidase-2 (3VAE) & -6.4 & [21] \\
\hline 14. & $\begin{array}{l}\text { Mmr-Multi-Drug Resistant Protein } \\
\text { (2IQ4) }\end{array}$ & -4.9 & [22] \\
\hline 15. & $\begin{array}{l}\text { Glf -UDP-Galactopyranose Mutase } \\
(1 \mathrm{~V} 0 \mathrm{~J}))\end{array}$ & -4.1 & [23] \\
\hline 16. & $\begin{array}{l}\text { EmbC-Arabinosyl-Indolyl Acetyl } \\
\text { Inositol Synthase (3PTY) }\end{array}$ & -3.1 & {$[24]$} \\
\hline D & \multicolumn{3}{|l|}{ Function (Regulatory Protein) } \\
\hline 17. & PknG-Protein Kinase (2PZI) & -8.2 & [25] \\
\hline
\end{tabular}

Table 1: Molecular docking results of Thioridazine from Glide [9].<smiles>COc1ccc2c(c1)N(CCC1CCCCN1C)c1ccccc1S2</smiles>

Figure 1: Thioridazine (Psychoactive and antitubercular drug).

\section{Discussion}

Comparative docking analysis of $\mathrm{TZ}$ with various pathophysiological enzymes responsible for TB is a reasonable method to study the TZ as a anti-TB drug. The TZ is reported for its efficacy towards TB in a curious mechanism, the docking results showed that it may act on other mechanisms possibly. The binding efficiency of the drug TZ with 17 pathophysiological enzymes (Table 1) and the G-Score have ranged between -3.1 to -9.5. The non-bonding interactions/hydrophobic region include the key amino acid residues like tyrosine (TYR), isoleucine (ILE), leucine (LEU) and phenylalanine (PHE) in top scored three enzymes against TZ. The TZ docked well with the enzymes Cyclopropane Mycolic Acid Synthase (lipid metabolism), Methoxy Mycolic Acid Synthase (lipid metabolism), NADH-Dependent Enoyl ACP (Acyl Carrier Protein) Reductase (lipid metabolism), and Protein Kinase-G (Regulatory Protein) with the G-Score of $-9.5 \mathrm{Kcal} / \mathrm{mol},-9.4 \mathrm{Kcal} / \mathrm{mol},-8.4 \mathrm{Kcal} / \mathrm{mol}$ and -8.2 $\mathrm{Kcal} / \mathrm{mol}$ respectively. This is an indication for an antidepressant drug TZ, against $M t b$ through an unusual mechanism has encouraged the study [9].

\section{Conclusion}

Thioridazine (TZ) as an antidepressant is reported to act by inhibiting the efflux pump mechanism. The docking studies indicated that it might be acting by inhibition of several enzymes notably Cyclopropane Mycolic Acid Synthase (lipid metabolism), Methoxy Mycolic Acid Synthase (lipid metabolism), NADH-Dependent Enoyl ACP (Acyl Carrier Protein) Reductase (lipid metabolism), and Protein Kinase-G (Regulatory Protein) with the G-Score of $-9.5 \mathrm{Kcal} /$ $\mathrm{mol},-9.4 \mathrm{Kcal} / \mathrm{mol},-8.4 \mathrm{Kcal} / \mathrm{mol}$ and $-8.2 \mathrm{Kcal} / \mathrm{mol}$ respectively. So the Anti-TB activity of TZ is not because of Efflux pump mechanism but may be by more than one mechanism possibly. The computational studies also concluded that the novel approach on TZ derivatives may lead to newer and more effective anti-TB drugs.

\section{Acknowledgement}

The authors are highly thankful to Department of Pharmaceutical chemistry, Himalayan Institute of Pharmacy Research, Dehradun, (Uttarakhand), India for providing facility and supports.

\section{Bibliography}

1. Global tuberculosis report World Health Organization (WHO), Geneva, Switzerland (2014): 8-10.

2. Udwadia ZF., et al. "Safety and efficacy of Thioridazine as salvage therapy in Indian patients with XDR-TB". Recent Patents on Anti-Infective Drug Discovery 6 (2011): 88-91.

3. Ordway., et al. "Clinical Concentrations of Thioridazine Kill Intracellular Multidrug-Resistant Mycobacterium tuberculosis". Journal of Antimicrobial Chemotherapy 47 (2003): 917-922. 
4. Amaral L and Molnar J. "Why and how Thioridazine in combination with antibiotics to which the infective strain is resistant will cure totally drug-resistant tuberculosis". Expert Review of Anti-infective Therapy 10 (2012): 869-873.

5. Leonard A., et al. "Activity of phenothiazines against antibiotic resistant Mycobacterium tuberculosis: a review supporting further studies that may elucidate the potential use of Thioridazine as antituberculosis therapy". Journal of Antimicrobial Chemotherapy 47 (2001): 505-511.

6. Leonard A., et al. "Thioridazine cures extensively drug-resistant tuberculosis (XDR-TB) and the need for global trials is now". International Journal of Antimicrobial Agents 35 (2010): 524-526.

7. Van Ingen J. "The broad-spectrum antimycobacterial activities of phenothiazines, in vitro: somewhere in all of this there may be patentable potentials". Recent Patents on Anti-Infective Drug Discovery 6 (2011): 104-109.

8. Dutta NK., et al. "New patentable use of an old neuroleptic compound Thioridazine to combat tuberculosis: a gene regulation perspective". Recent Patents on Anti-Infective Drug Discovery 6 (2011): 128-138.

9. Suresh R., et al. "Insights into thioridazine for its anti-tubercular activity from molecular docking studies". International Journal of Pharmacy and Pharmaceutical Sciences 7 (2015): 344-346.

10. Daniel B., et al. "Mycolic acid cyclopropanation is essential for viability, Drug Resistance, and Cell Wall Integrity of Mycobacterium tuberculosis". Chemical Biology 16 (2009): 499-509.

11. Michael SG. "The mmaA2 Gene of Mycobacterium tuberculosis Encodes the Distal Cyclopropane Synthase of the $\alpha$-Mycolic Acid". Journal of Biological Chemistry 278 (2003): 7844-7849.

12. Xin H., et al. "Inhibition of the Mycobacterium tuberculosis enoyl acyl carrier protein reductase InhA by arylamides". Bioorganic and Medicinal Chemistry 15 (2007): 6649-6658.

13. Musayev F., et al. "Crystal structure of a substrate complex of Mycobacterium tuberculosis-ketoacyl-acyl carrier protein synthase III (FabH) with lauroyl-coenzyme A". Journal of Molecular Biology 346 (2005): 1313-1321.

14. Qiao C., et al. "O-[(N-Acyl) sulfamoyl] adenosines as antitubercular agents that inhibit mbta: an adenylation enzyme required for siderophore biosynthesis of the mycobactins". Journal of Medicinal Chemistry 50 (2007): 6080-6094.
15. Kremer L., et al. "Biochemical characterization of acyl carrier protein (AcpM) and Malonyl-CoA: AcpM Transacylase (mtFabD), Two Major Components of Mycobacterium tuberculosis fatty acid synthase II". Journal of Biological Chemistry 276 (2001): 27967-27974.

16. Huang CC., et al. "Crystal structures of Mycolic acid Cyclopropane synthases from Mycobacterium tuberculosis". Journal of Biological Chemistry 277 (2002): 11559-11569.

17. Vadim M., et al. "Benzothiazinones Kill Mycobacterium tuberculosis by blocking arabinan synthesis". Science 324 (2009): 801-804.

18. William BP., et al. "Identification and characterization of a unique adenosine kinase from Mycobacterium tuberculosis". Journal of Bacteriology 183 (2003): 6548-6555.

19. Nordqvist A., et al. "Synthesis, Biological evaluation and x-ray crystallographic studies of imidazo (1, 2-A) pyridine-based Mycobacterium Tuberculosis Glutamine Synthetase Inhibitors". Med Chem Comm 3 (2012): 620.

20. Sampathkumar P., et al. "Structure of Mycobacterium tuberculosis flavin dependant Thymidylate Synthase (MtbThyX) at 2.0 $\mathrm{A}^{0}$ resolution". Journal of Molecular Biology 352 (2003): 10911104.

21. Sierra LD., et al. "X-ray Crystal Structure of TMP Kinase from Mycobacterium tuberculosis complexed with TMP at $1.95 \mathrm{~A}^{0}$ resolution". Journal of Molecular Biology 311 (2001): 87-100.

22. Liliana R., et al. "Role of the mmr efflux pump in drug resistance in Mycobacterium tuberculosis". Journal of Antimicrobe Chemotherapy 57 (2013): 751-757.

23. Pan F., et al. "Cell wall core galactofuran synthesis is essential for growth of mycobacteria". Journal of Bacteriology 183 (2001): 3991-3998.

24. Belanger AE., et al. "The embA, B genes of Mycobacterium avium encode an arabinosyl transferase involved in cell wall arabinan biosynthesis that is the target for the antimycobacterial drug ethambutol". PNAS 93 (1996): 11919-11924.

25. Nicole S., et al. "Structural basis for the specific inhibition of protein kinase G, a virulence factor of Mycobacterium tuberculosis". PNAS 104 (2007): 12151-12156.

\section{Volume 2 Issue 11 November 2019 (C) All rights are reserved by Mohammad Asif and Md Tauquir Alam.}

